\title{
TWO-DIMENSIONAL FOKKER-PLANCK MODELS
}

\author{
KOJI TAKAHASHI \\ Department of Earth and Space Science, \\ Faculty of Science, Osaka University, \\ Toyonaka, Osaka 560, Japan
}

\begin{abstract}
.
The evolution of spherical single-mass star clusters was followed by numerically solving the orbit-averaged two-dimensional Fokker-Planck equation in energy-angular momentum space. Velocity anisotropy is allowed in the two-dimensional Fokker-Planck model. The development of the anisotropy is discussed in detail.
\end{abstract}

\section{Introduction}

Now, direct numerical integration of the orbit-averaged Fokker-Planck (hereafter FP) equation is a main tool to study the dynamical evolution of globular clusters. A direct-integration scheme was invented by Cohn (1979, 1980). Cohn (1979) first performed direct numerical integration of the time-dependent two-dimensional (hereafter 2D) FP equation in energyangular momentum $(E, J)$ space with the calculation of a self-consistent potential. Although Cohn (1979) showed the potential power of the directintegration scheme, he had to stop the calculation at a relatively early stage of gravothermal core collapse due to a numerical error concerning energy conservation; the central density of the cluster had increased by only three orders of magnitude, when the calculation was stopped. Later, Cohn (1980) assumed isotropy of the velocity distribution and treated the onedimensional (hereafter 1D) energy-space FP equation. The use of the 1D FP equation has some advantages over the use of the 2D FP equation: one of these is that it saves a lot of computation time and computer memory space; another one is that the numerical error in energy conservation is greatly reduced (Cohn 1980). This reduction of the error is largely due to 
the adoption of Chang and Cooper's (1970) finite-differencing scheme for the 1D FP equation. Cohn (1980) reported that the secular energy drift rate was reduced by better than a factor of 100 by adopting of the ChangCooper scheme. In fact, he could follow the core collapse until the central density increased by twenty orders of magnitude.

Because of those advantages of the 1D FP equation, the 1D FP equation has been used in most FP studies concerning globular-cluster evolution, while the 2D FP equation has seldom been used. The adoption of the simpler 1D (isotropic) FP equation seems to be quite reasonable for studying the core evolution, which has been a main subject during the past two decades, because strong relaxation enforces the isotropy of the velocity distribution in the core.

On the other hand, it is true that the development of anisotropy in the halo is a natural consequence of cluster evolution driven by two-body relaxation. The relaxation is strong in the core because of its high density, and the strong relaxation continues to produce high-energy stars. Such highenergy stars have very radial orbits on the average, and they travel through the low-density halo almost without experiencing collisions. Therefore, radial orbits predominate in the halo and the velocity anisotropy increases as the halo grows. The penetration of anisotropy even into the inner region was pointed out in several early studies (e.g., Cohn 1985). This is closely related to gravothermal core collapse.

Thus, the development of velocity anisotropy is expected to occur throughout the whole cluster. In fact, so far, anisotropy has been considered in various simulations concerning the evolution of star clusters (see Takahashi 1995a). Recently, furthermore, more elaborate anisotropic gaseous and higher-order fluid-dynamical models of star clusters have been developed (e.g., Bettwieser and Spurzem 1986; Louis 1990; Louis and Spurzem 1991; Spurzem 1991; Giersz and Spurzem 1994; Spurzem and Takahashi 1995). They have made it possible to calculate in detail the evolution of anisotropic star clusters. On the other hand, more fundamental 2D FP simulations were carried out only for isolated single-mass clusters without binaries (Cohn 1979, 1985).

Considering the above situations, I think that it is now a good time to reconsider direct 2D FP calculations. Concerning the practical sides of computations, it is now possible to carry out the 2D calculations on standard workstations. A main obstacle to the 2D FP calculations is to develop computational schemes of high accuracy (especially in the energy conservation). The aim of this work is to develop reliable numerical schemes for the 2D orbit-averaged FP equation and to consider in detail the evolution of anisotropic star-clusters. More complete descriptions of the present topic are found in Takahashi (1995a, pre-collapse evolution; 1995b, post-collapse 
evolution).

\section{The Orbit-Averaged Fokker-Planck Equation}

We consider the evolution of spherical one-component star clusters. In a steady-state spherical system, the distribution function $f(\mathbf{r}, \mathbf{v}, t)$ is a function of only the energy $E$ and total angular momentum $J$ per unit mass. The evolution of $f$ due to two-body relaxation can be described by the orbit-averaged FP equation in $(E, J)$-space (Cohn 1979). The 2D FP equation under the fixed gravitational potential $\phi(r)$ can be written in a fluxconserving form,

$$
A \frac{\partial f}{\partial t}=-\frac{\partial F_{E}}{\partial E}-\frac{\partial F_{R}}{\partial R}
$$

where

$$
\begin{aligned}
& -F_{E}=D_{E E} \frac{\partial f}{\partial E}+D_{E R} \frac{\partial f}{\partial R}+D_{E} f, \\
& -F_{R}=D_{R E} \frac{\partial f}{\partial E}+D_{R R} \frac{\partial f}{\partial R}+D_{R} f .
\end{aligned}
$$

Here, $R$ is the scaled angular-momentum: $R=J^{2} / J_{\mathrm{c}}^{2}(E)$, where $J_{\mathrm{c}}(E)$ is the angular momentum of a circular orbit of energy $E$. Thus, $R$ takes all values between 0 and 1 , independent of $E$. The isotropized distribution function, $\bar{f}(E, r)$, introduced by Cohn (1979) was used to calculate the diffusion coefficients, $D_{E E}, D_{E}$, etc. The isotropization makes computing the coefficients much easier. It has generally been conceived that the use of the isotropized distribution function does not cause significant errors, because the coefficients only depend on the moments of $f(E, R)$, and because relaxation occurs mainly in the core where the distribution function is almost entirely isotropic. However, as shown below, since $f(E, R)$ strongly depends on $R$ for $E \sim 0$ as the halo develops, we may have to be more careful about the use of the isotropized distribution function.

\section{The Method}

The framework of our method is the same as that of Cohn's (1979) method. Cohn's method comprises two steps: the FP step and the Poisson step. In the FP step, the distribution function is advanced by solving the FP equation with the gravitational potential being held fixed. In the Poisson step, the potential is advanced by solving Poisson's equation with the distribution function being held fixed as a function of the adiabatic invariants.

An essential difference between our method and Cohn's method exists only concerning a discretization scheme of the FP equation. Two different discretization schemes have been developed: one is a finite-difference 
scheme where the Chang-Cooper scheme is simply applied for only the energy direction; the other is a finite-element scheme where the test and weight functions suggested by the generalized variational principle (Inagaki and Lynden-Bell 1990) are used. The details concerning the discretization schemes are described in Takahashi (1995a).

The FP equation is solved in a rectangular domain enclosed by boundary lines, $E=\phi(0), E=E_{\min }, R=0$, and $R=1$, where $\phi(0)$ is the central potential and the value of $E_{\min }$ is chosen to be close to zero. We impose boundary conditions that $F_{E}=0$ on boundaries $E=\phi(0), E_{\min }$, and $F_{R}=$ 0 on boundaries $R=0,1$. We use variables $(X, Y)$ instead of $(E, R)$ in practical calculations. The variable $X(E)$ is defined by

$$
X(E)=\ln \left[\frac{E}{2 \phi(0)-E_{0}-E}\right]
$$

where $E_{0}$ is an adjustable parameter (Cohn 1979). The variable $Y(R)$ is defined by

$$
Y(R)=\frac{\ln \left(1+R / R_{0}\right)}{\ln \left(1+1 / R_{0}\right)}
$$

where $R_{0}$ is an adjustable parameter, such that $0<R_{0} \ll 1$. We set $R_{0}=0.01$ in standard runs. This variable is introduced in order to give a good representation to radial orbits.

\section{Results}

Calculations were carried out using both the finite-difference and finiteelement codes. The results obtained by the two (partially) different codes were generally in good agreement. For calculations of the pre-collapse evolution, the numerical accuracy of the two schemes is similar. However, for calculations of the post-collapse evolution, the accuracy (in particular, concerning total-energy conservation) of the finite-difference scheme is better. The reason why the accuracy of the finite-element scheme is not very good for the post-collapse calculations is not clear at present. The figures shown below were actually drawn from the results of calculations by the finitedifference code.

The initial condition of the calculations was Plummer's model, where the velocity distribution is isotropic everywhere. We use standard units such that $G=M=1$ and $\mathcal{E}_{\mathrm{i}}=-1 / 4$, where $G$ is the gravitational constant, $M$ is the total mass, and $\mathcal{E}_{\mathrm{i}}$ is the initial total energy.

\subsection{PRE-COLLAPSE EVOLUTION}

Unless any heat sources are included in FP calculations, the core continues to contract and the core density continues to increase [gravothermal core 
collapse (Lynden-Bell and Eggleton 1980)]. A calculation was continued until the central density increased by about 14 orders of magnitude. During the calculation, the numerical error in the total mass was within $0.1 \%$, and the error in the total energy was within $1 \%$.
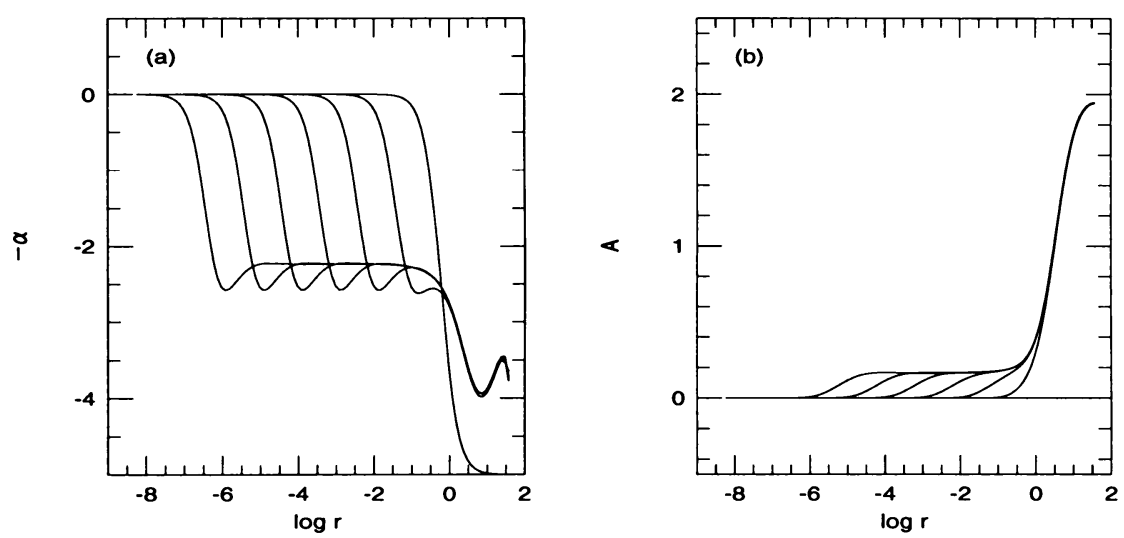

Figure 1. (a) Evolution of the radial profile of the logarithmic density gradient, $d \ln \rho / d \ln r=-\alpha$. The power-law region with $\alpha=2.23$ extends into the inner region as the core collapse proceeds. (b) Evolution of the radial profile of the anisotropy parameter, $A \equiv 2-2 \sigma_{\mathrm{t}}^{2} / \sigma_{\mathrm{r}}^{2}$. The self-similar region with $A=0.16$ extends into the inner region as the core collapse proceeds.

Figure 1a shows the evolution of the radial profile of the logarithmic density gradient, $d \ln \rho / d \ln r=-\alpha$. The power-law region with $\alpha=2.23$ extends into the inner region self-similarly as the core collapse proceeds. This value of $\alpha=2.23$ coincides with that found in the 1D (isotropic) FP model (Cohn 1980; Heggie and Stevenson 1988). Figure 1b shows the evolution of the radial profile of the anisotropy parameter, $A \equiv 2-2 \sigma_{\mathrm{t}}^{2} / \sigma_{\mathrm{r}}^{2}$, where $\sigma_{\mathrm{r}}$ and $\sigma_{\mathrm{t}}$ are the radial and (1D) tangential velocity dispersions, respectively. The self-similar region with $A=0.16$ (or $\sigma_{\mathrm{t}}^{2} / \sigma_{\mathrm{r}}^{2}=0.92$ ) extends into the inner region as the core collapse proceeds. Cohn's (1985) calculation gave a similar value of $A \approx 0.15$. However, the degree of anisotropy in the halo is somewhat stronger in our calculation than in Cohn's calculation.

At late stages of the core collapse, the collapse rate $\xi \equiv t_{\mathrm{r}}(0) d \ln \rho(0) / d t$, where $t_{\mathrm{r}}(0)$ is the central relaxation time (Spitzer and Hart 1971a) and $\rho(0)$ is the central density, tends to an asymptotic constant value of $\xi=2.9 \times$ $10^{-3}$. On the other hand, Cohn's (1979) $2 \mathrm{D}$ calculation gave $\xi=6.0 \times 10^{-3}$ and Cohn's (1980) $1 \mathrm{D}$ calculation did $\xi=3.6 \times 10^{-3}$. While the value of $\xi$ in our anisotropic model is smaller than that in the isotropic model, the value of $\xi$ in Cohn's (1979) anisotropic model is larger. Which tendency 
is true? I think that Cohn's value is less reliable than our value, because Cohn's calculation was accompanied by a large error in energy conservation $(\sim 11 \%)$ and did not follow the core collapse very deeply. Although I cannot give satisfactory proof of the slower collapse in anisotropic clusters, the following intuitive interpretation may be helpful: the reduction of the 2D FP equation to the 1D FP equation by averaging over angular momentum space causes artificial diffusion in addition to real diffusion, and, consequently, the core collapse proceeds faster in isotropic clusters. It is interesting that Louis (1990) also found the lower value of $\xi$ in anisotropic clusters using fluid-dynamical models.

Figure 2a shows the evolution of Lagrangian radii for the $2 \mathrm{D}$ and $1 \mathrm{D}$ models. The time is measured in units of the initial half-mass relaxation time $t_{\mathrm{rh}, \mathrm{i}}$ (Spitzer and Hart 1971a). We find again slower core collapse in the $2 \mathrm{D}$ calculation from figure $2 \mathrm{a}$. The $2 \mathrm{D}$ calculation gives a core collapse time of $t_{\mathrm{coll}}=17.6 t_{\mathrm{rh}, \mathrm{i}}$ and the $1 \mathrm{D}$ calculation gives $t_{\mathrm{coll}}=15.6 t_{\mathrm{rh}, \mathrm{i}}$. Figure $2 \mathrm{~b}$ also shows the evolution of Lagrangian radii, but the time for the $1 \mathrm{D}$ calculation is scaled so that the collapse time in the $1 \mathrm{D}$ calculation should coincide with that in the 2D calculation. In this figure, we do not find any significant differences between the two models for the 1-75\% Lagrangian radii. However, the $90 \%$ radius of the $2 \mathrm{D}$ model expands further than that of the $1 \mathrm{D}$ model; that is, the $2 \mathrm{D}$ model has a more extended halo.
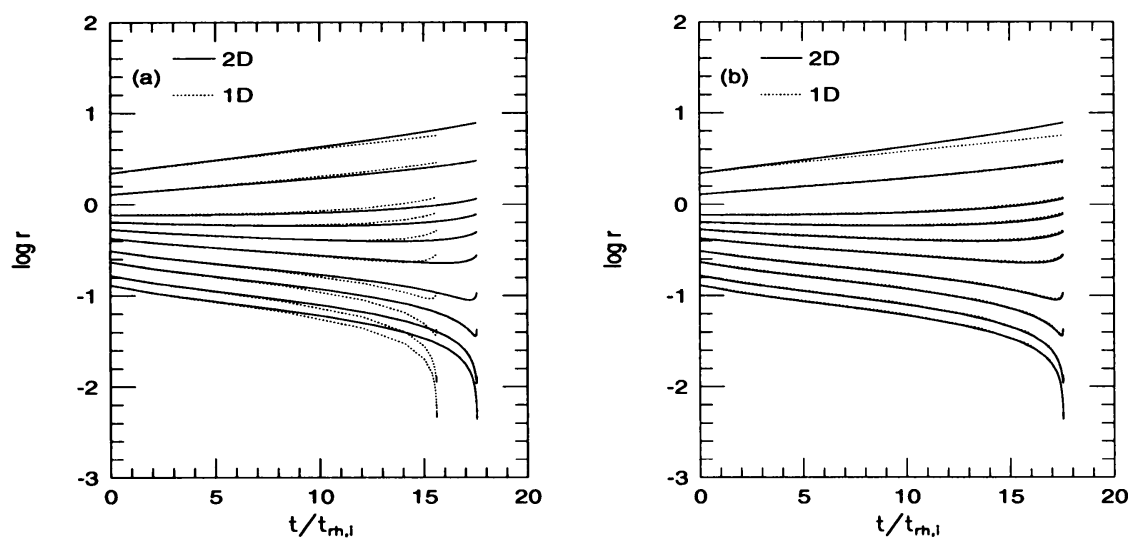

Figure 2. (a) Evolution of Lagrangian radii containing inner 1, 2, 5, 10, 20, 30, 40, 50, 75 , and $90 \%$ of the cluster mass. The solid curves are the result of the $2 \mathrm{D}$ FP calculation, while the dotted curves are that of $1 \mathrm{D}$ calculation. The time is measured in units of the initial half-mass relaxation time $t_{\mathrm{rh}, \mathrm{i}}$. (b) Same as (a), but the time axis of $1 \mathrm{D}$ calculation is multiplied by a constant factor so that the collapse time in the 1D calculation should coincide with that in the $2 \mathrm{D}$ calculation. 

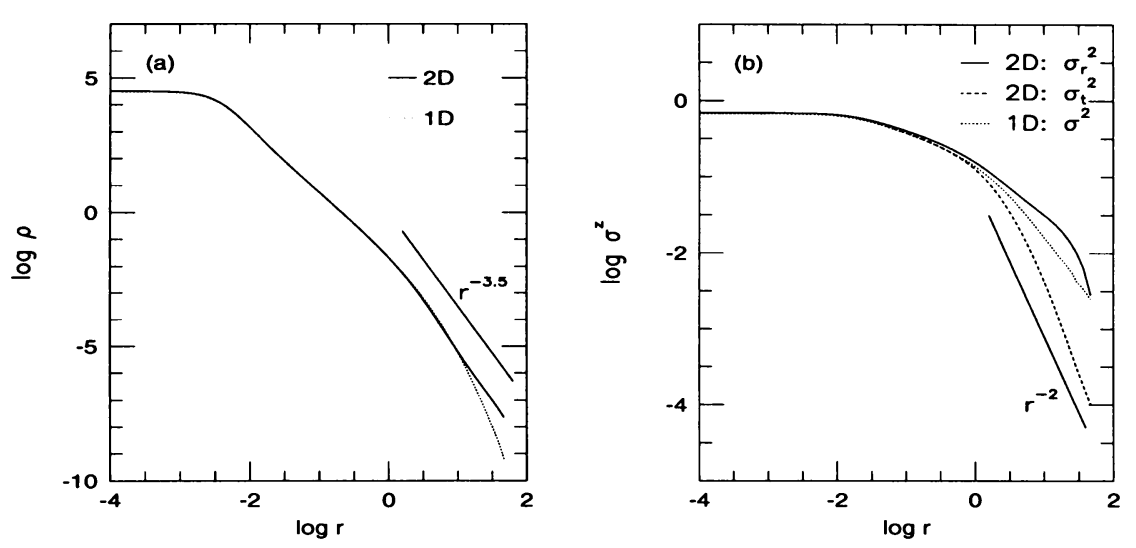

Figure 3. (a) Density profile at an epoch when the central density increases by about 4.5 orders of magnitude for the 2D model (the solid curve), and for the 1D model (the dotted curve). The asymptotic line $\rho \propto r^{-3.5}$ is shown for a comparison. (b) Velocity dispersion profiles at the same epochs as in (a). The solid and dashed curves are the radial and 1D tangential velocity dispersions for the $2 \mathrm{D}$ model, respectively. The dotted curve is the $1 \mathrm{D}$ velocity dispersion profile for the $1 \mathrm{D}$ model. The asymptotic line $\sigma^{2} \propto r^{-2}$ is shown for a comparison.

Figure 3a shows the density profiles at epochs when the central density increases by about 4.5 orders of magnitude for the $2 \mathrm{D}$ and $1 \mathrm{D}$ models. The density profile in the outer halo is approximated by a power law, $\rho \propto$ $r^{-3.5}$, rather well for the 2D model (cf. Spitzer and Shapiro 1972). In figure $3 \mathrm{~b}$, the velocity dispersion profiles at the same epochs as in figure $3 \mathrm{a}$ are shown. In the halo the radial velocity dispersion exceeds the tangential velocity dispersion considerably. This is because the halo is dominated by eccentric orbits. A power law, $\sigma_{\mathrm{t}}^{2} \propto r^{-2}$, gives a reasonable fit to the result of the 2D FP calculation in the halo. This power law corresponds to the constant mean squared angular momentum (cf. Spitzer and Hart 1971b; Hénon 1971).

\subsection{POST-COLLAPSE EVOLUTION}

Now we take account of heating effects by three-body binaries. The threebody binary heating rate per unit mass is given by

$$
\dot{E}_{\mathrm{b}}=C_{\mathrm{b}} G^{5} m^{3} \rho^{2} \sigma^{-7}
$$

where $C_{\mathrm{b}}$ is a numerical coefficient (Hut 1985). A standard value of $C_{\mathrm{b}}=90$ was chosen in the present calculations. The local heating rate (5) is orbitaveraged (Cohn 1979), and then the orbit-averaged heating rate, $\left\langle\dot{E}_{\mathrm{b}}\right\rangle_{\text {orb }}$, 
is added to the usual first-order diffusion coefficient $\langle\Delta E\rangle_{\text {orb }}$. Furthermore, we assume that binary scatterings do not produce a net change of the scaled angular momentum $R$, i.e. $\left\langle\dot{R}_{\mathrm{b}}\right\rangle_{\text {orb }}=0$.

For calculations of the post-collapse evolution, we must specify the number of stars in the cluster, $N$, and the numerical constant, $\mu$, in the Coulomb $\operatorname{logarithm} \ln (\mu N)$. For all the present calculations, the value of $\mu=0.11$ was chosen, which was suggested by Giersz and Heggie (1994a) for the precollapse evolution of single-mass clusters. Calculations were performed for $N=5000,10000$, and 20000. Concerning computation time, for example, the 2D FP calculation for $N=20000$, where $151 X$-mesh, $35 Y$-mesh and $91 r$-mesh points were used, required about 95 hours of CPU time on HP $9000 / 715(50 \mathrm{MHz})$.

The core expansion is stable for $N=5000$, marginally stable (overstable) for $N=10000$. For $N=20000$, the core expansion is unstable: the central density oscillates chaotically with the large amplitude [gravothermal oscillation (Bettwieser and Sugimoto 1984)]. There are no qualitative difference concerning the features of the gravothermal oscillations between $1 \mathrm{D}$ and $2 \mathrm{D}$ calculations. Figures $4 \mathrm{a}$ and $4 \mathrm{~b}$ show the evolution of the anisotropy $A$ averaged over $0-1 \%, 1-2 \%, \ldots$, and $75-90 \%$ Lagrangian radii, for the case of $N=20000$. The anisotropy at inner Lagrangian radii oscillates with the core oscillation. We can see the anisotropy oscillation even at the half-mass radius.
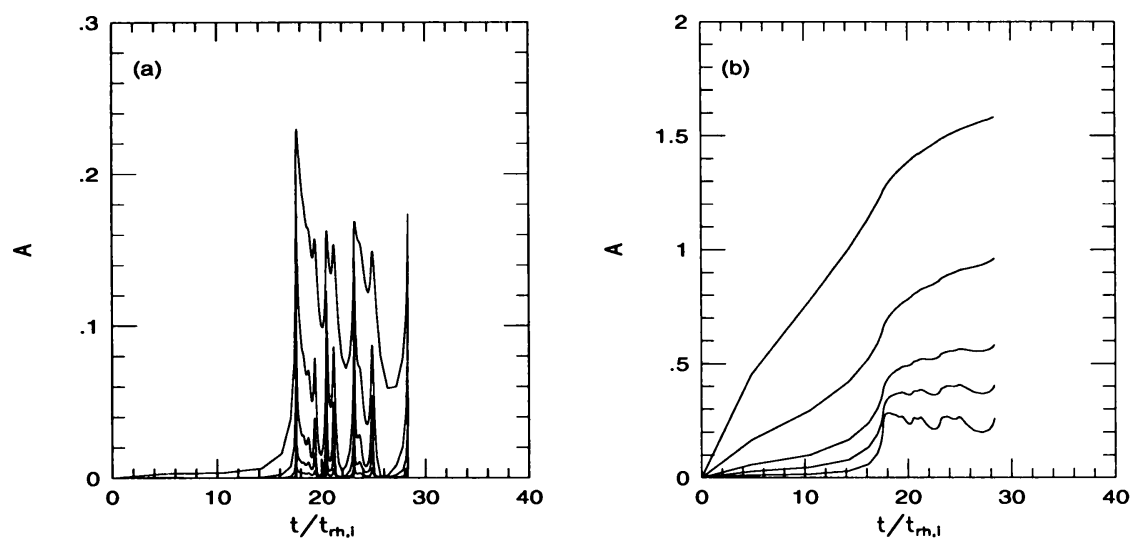

Figure 4. (a) Evolution of the anisotropy $A$ averaged over $0-1 \%, 1-2 \%, 2-5 \%, 5-10 \%$, and 10-20\% Lagrangian radii, for the case of $N=20000$. (b) Same as (a), but for the anisotropy $A$ averaged over $20-30 \%, 30-40 \%, 40-50 \%, 50-75 \%$, and $75-90 \%$ Lagrangian radii. $A$ increases as the radius increases. 

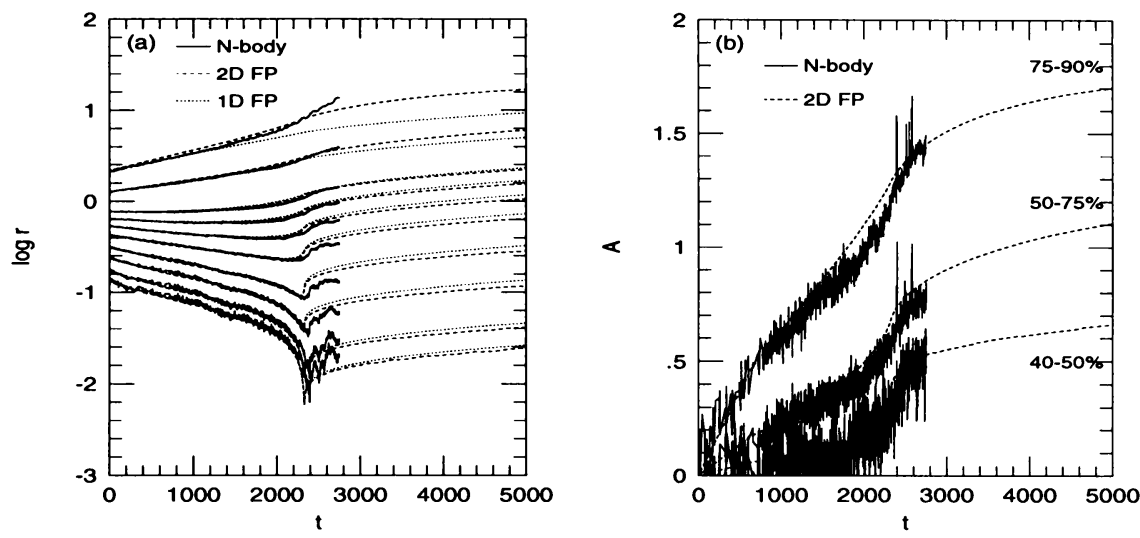

Figure 5. (a) Evolution of the 1, 2, 5, 10, 20, 30, 40, 50, 75, and 90\% Lagrangian radii for $N=10000$. The solid curves are the result of the $N$-body calculation (by R. Spurzem), the dashed curves are that of the 2D FP calculation, and the dotted curves are that of the 1D FP calculation. The time for the 1D calculation is scaled as in figure 2b. (b) Evolution of the anisotropy $A$ averaged over the $40-50 \%, 50-75 \%$, and $75-90 \%$ Lagrangian radii. The solid curves are the result of the $N$-body calculation, and the dashed curves are that of the 2D FP calculation.

Lastly, we compare the FP models and an $N$-body model for $N=10000$. The result of the 10000-body calculation has kindly been made available by Rainer Spurzem (cf. Spurzem and Aarseth 1995). Figure 5a shows a comparison of the evolution of Lagrangian radii. The time for the $1 \mathrm{D}$ calculation is scaled as in figure $2 \mathrm{~b}$. The result is generally in good agreement between the 2D FP and $N$-body models. In particular, the collapse times for the two models are very close. However, we should remember that the collapse time in physical time units in the FP model depends on $\mu$, and that there is a scatter of collapse times for each individual $N$-body simulation (Giersz and Heggie 1994a, b; Spurzem and Aarseth 1995). The good agreement of the collapse time in this case may be accidental. In the $N$-body model, the inner Lagrangian radii oscillate with the small amplitude after the core bounce. This may be due to stochastic binary activities. Figure 5b shows the evolution of the anisotropy $A$ in the outer regions. The agreement between the 2D FP and $N$-body models is good. This fact supports the reliability of the present 2D FP model.

\section{Conclusions and Discussion}

I have developed numerical codes to solve the orbit-averaged $(E, J)$-space FP equation with high accuracy, and have investigated in detail the pre- and 
post-collapse evolution of single-mass spherical star clusters where velocity anisotropy is allowed. Two different integration schemes have been developed: they are the finite-difference scheme and the finite-element scheme. Using these schemes, we could follow the core collapse until the central density increased by about 14 orders of magnitude, and follow long-term evolution past the core collapse.

We have seen that the relaxation process is always accompanied by the velocity anisotropy production. We still do not know very much the effects of anisotropy on the evolution of more realistic clusters (e.g., multimass clusters, tidally limited clusters, etc.). Since the present work has shown that 2D FP calculations can be performed with reasonable numerical accuracy, we do not have to hesitate doing 2D FP calculations from now on. The evolution of more realistic cluster models will be studied in the future.

The author is a research fellow of the Japan Society for the Promotion of Science, and this work was supported in part by the Grand-in-Aid for Encouragement of Young Scientists by the Ministry of Education, Science and Culture of Japan (No. 1338).

\section{References}

Bettwieser E., Spurzem R. 1986, A\&A 161, 102

Bettwieser E., Sugimoto D. 1984, MNRAS 208, 493

Chang J.S., Cooper G. 1970, J. Comp. Phys. 6, 1

Cohn H. 1979, ApJ 234, 1036

Cohn H. 1980, ApJ 242, 765

Cohn H. 1985, in Dynamics of Star Clusters, IAU Symp No.113, ed J. Goodman, P. Hut (D. Reidel Publishing Company, Dordrecht) p161

Giersz M., Heggie D.C. 1994a, MNRAS 268, 257

Giersz M., Heggie D.C. 1994b, MNRAS 270, 298

Giersz M., Spurzem R. 1994, MNRAS 269, 241

Heggie D.C., Stevenson D. 1988, MNRAS 230, 223

Hénon M. 1971, Ap\&SS 13, 284

Hut P. 1985, in Dynamics of Star Clusters, IAU Symp No.113, ed J. Goodman, P. Hut

(D. Reidel Publishing Company, Dordrecht) p231

Inagaki S., Lynden-Bell D. 1990, MNRAS 244, 254

Louis P.D. 1990, MNRAS 244, 478

Louis P.D., Spurzem R. 1991, MNRAS 251, 408

Lynden-Bell D., Eggleton P.P. 1980, MNRAS 191, 483

Spitzer L.Jr., Hart M.H. 1971a, ApJ 164, 399

Spitzer L.Jr., Hart M.H. 1971b, ApJ 166, 483

Spitzer L.Jr., Shapiro S.L. 1972, ApJ 173, 529

Spurzem R. 1991, MNRAS 252, 177

Spurzem R., Aarseth S.J., 1995, in preparation

Spurzem R., Takahashi K. 1995, MNRAS 272, 772

Takahashi K. 1995a, PASJ 47, in press

Takahashi K. 1995b, in preparation 